\title{
Ant colony system algorithm for optimal network reconfiguration
}

\author{
Shabana Mehfuz \\ Department of Electrical Engineering, Jamia Millia Islamia \\ New Delhi-110025, India \\ E-mail:mehfuz_shabana@yahoo.com \\ Farah Rashid \\ Department of Electrical Engineering, Jamia Millia Islamia \\ New Delhi-110025, India \\ E-mail:83.farah@gmail.com
}

Received 21 May 2013

Accepted 18 March 2014

\begin{abstract}
Network reconfiguration of a power distribution system is an operation to alter the topological structure of distribution feeders by changing open/closed status of sectionalizing and tie switches. Network reconfiguration balances feeder loads and helps in managing overload conditions of the network by transferring load from heavily loaded feeders to lightly loaded ones. In this paper we have introduced an ant colony system algorithm for performing network reconfiguration efficiently so as to minimize power losses occurring in a distribution network. The main idea is that of having a set of agents, called ants, which perform search in parallel for good solutions and cooperate through pheromone-mediated indirect and global communication. Informally, each ant constructs a solution path in an iterative way. Validation of the proposed algorithm has been carried using a standard IEEE network. The results found are satisfactory and prove ant colony system algorithm to be an efficient tool for optimal network reconfiguration.
\end{abstract}

Keywords : Optimal losses, Network reconfiguration, Load Flow, Ant Colony Optimization 


\section{Introduction}

Network reconfiguration of distribution networks is a very important tool that saves energy. Also, because of its features it is inherently an optimization problem ${ }^{1}$. The distribution systems are important links between the utility and the customer, in which sectionalizing switches are used for both protection and configuration management. Recent studies indicate that up to $13 \%$ of the total power generation is wasted in the form of line losses at the distribution level. Therefore, investigation of methods for network reconfiguration is of great benefit. The objective of network reconfiguration is to reduce power losses and improve the reliability of power supply by changing the status of existing sectionalizing switches and ties ${ }^{2}$.

Authors have ${ }^{3}$ conducted the early work on feeder reconfiguration for loss reduction. Another work defined the problem of loss reduction and load balancing as an integer-programming problem ${ }^{4}$. An implementation has been presented that used a genetic algorithm to achieve the minimum loss configuration $^{5}$. Authors ${ }^{6}$ have suggested the use of the power flow method based on a heuristic algorithm to determine the minimum loss configuration of radial distribution networks. The authors $^{7-10}$ have proposed a solution procedure that employed simulated annealing (SA) to search for an acceptable non- inferior solution. A methodology has been outlined and validated for optimizing the operation of megavolt (MV) distribution networks. The authors ${ }^{11}$ have considered time varying load analysis to reduce loss in their work.

Network reconfiguration is a combinatorial, complex, non-differentiable, constrained optimization problem because the distribution system involves many candidate-switching combinations $^{12}$. Although this problem has been solved by the above methods, either optimality is not guaranteed or much computation time is required. Differential evolution (DE) ${ }^{13-15}$ is one of the best evolution algorithms (EAs). DE is a method in which function parameters are encoded as floating-point variables and is based on stochastic searches ${ }^{16}$. This method has been verified as a promising candidate for solving real-valued optimization problems. However, it may be likely to reach a local optimum or result in premature convergence. This shortcoming could be overcome by employing a larger population, but to do so requires much computation time. A hybrid version of DE (called HDE) has been proposed ${ }^{17}$ to avoid the need for employing a large population. The hybrid version embeds two additional operationsacceleration phase and migration phase. The migration operation allows the HDE to upgrade the exploration of the search space and increase the likelihood of success of a global search. The best fitness may not descend continually from generation to generation. An acceleration operation can be applied to improve the fitness. However, a general mixed-integer nonlinear programming (MINLP) problem includes continuous and discrete variables and the $\mathrm{MIHDE}^{18,19}$ can be applied to handle this problem. Authors ${ }^{23}$ have suggested the fuzzy framework using adaptive genetic algorithm for multi-objective reconfiguration of radial distribution systems. In this the initial populations for genetic algorithm is created using a heuristic approach and the genetic operators are adapted with the help of graph theory to generate feasible individuals. Authors ${ }^{24}$ takes the distribution system reconfiguration as a nonlinear and multiobjective problem and suggests the node-depth encoding (NDE) which enables a modeling of Distribution System problems that eliminates several constraint equations from the usual formulation. A multiobjective evolutionary algorithm (EA) based on subpopulation tables is used to models several objectives and constraints, enabling a better exploration of the search space. Authors ${ }^{25}$ presents a genetic algorithm (GA) with two network encodings, capable of representing only radial connected solutions without demanding a planar topology or any specific genetic operator. The code was named sequential because the evaluation of the $i$ th gene depends on the information forthcoming from all the previous genes. This method requires external parameters such as cross over rate and mutation rate and it is hard to determine these parameters in prior. Authors ${ }^{26}$ uses a harmony search algorithm to solve the network reconfiguration problem to get optimal switching combination in the network which results in minimum loss. Authors ${ }^{27}$ uses a binary particle swarm optimization-based search algorithm to evaluate the reliability at the load points and formulate an algorithm to find the minimal set of components appearing between the feeder and any particular load point. Authors ${ }^{28}$ suggests a 
combination of Opposition based Differential Evolution Algorithm (ODE) and Plant Growth Simulation Algorithm (PGSA) to solve the optimization problem. The optimization approach based on PGSA provides a detailed description on switch states and ODE improves the efficiency of optimization by reducing the number of load flow executions. But all these methods are complicated in nature and in comparison ant colony system is found to be a much more simple and powerful tool for getting an optimal network reconfiguration.

In this paper, an ACS algorithm network reconfiguration is solved by means of ant colony system algorithm.

Section 2 provides details of ant colony system. Section 3 gives the description of the problem and the proposed ACS algorithm for network reconfiguration. Section 4 talks about the implementation. Section 5 discusses the results. In section 6 , brief conclusions are given.

\section{Ant Colony System Optimization}

The behavior of the real ant colony is the motivation behind the ACO algorithms ${ }^{20}$. The algorithm can find the optimum solution by generating artificial ants. As the real ants search their environment for food, the artificial ants search the solution space. The probabilistic movement of ants in the system allows the ants to explore new paths and to re-explore the old visited paths. An important insight of early research on ant's behavior was that most of the communication among individuals and the environment is based on the use of chemicals produced by ants. These chemicals are called pheromones. The strength of the pheromone deposit directs the artificial ants toward the best paths and the pheromone evaporation allows the system to forget old information and avoid quick convergence to sub optimal solutions, which is the overall result of the Ant's repeated and concurrent path sampling experiences provides the local intensity of the pheromone field ${ }^{21}$. It is encoded as a spatially distributed measure of goodness associated with each move. This indirect communication among agents locally modifies the environment and reacts to these modifications too, forms a distributed control. This control leading to a phase of global coordination of the agent actions is called stigmergy. Stigmergic coordination leads to the self-organized behaviors not only in ant colonies but more generically across social systems, from insects to humans. When stigmergic coordination takes place, system's protocols (interfaces) play a prominent role with respect to modules (agents). Protocols are the rules that prescribe the characteristics of the allowed interfaces and of the information exchanged between modules. This permits system functions that could not be achieved by isolated modules. A stigmergic model which supplies global robustness, scalability, evolvability, and allows to fully exploit the potentialities of the modules and of modularity is supposed to be good. In Fig. 1(a) ants are looking for food starting at the ant-hill with two different paths available. Every ant deposits a pheromone trail. The pheromone deposited by the ants taking the same path is added. After loading food, the ants go back the path they came, while the ones that accidentally took the shorter path will return to the ant-hill earlier. In Fig. 1(b) the blue circles mark the ants that took the shorter path. Because they also deposit pheromone on their way back, the shorter path gets a higher concentration of pheromone than the longer one, on which the returning ants are not on the way back to the ant-hill yet. Following ants will favor the path with the higher pheromone concentration and therefore more and more ants will choose to use the shorter path. Finally, the longer path is not used any more and the ant colony has optimized food winning as shown in Fig.

\section{Problem Description \& ACS Algorithm}

The main objective of a feeder reconfiguration during any undesirable condition that exists in the network, is to find the network, which is having economical losses ${ }^{22}$. Therefore the probability $\left(\mathrm{p}^{\mathrm{k}}{ }_{\mathrm{ij}}\right)$ between node $\mathrm{i}$ and $\mathrm{j}$ depends on two functions Pheromone intensity and Heuristic function which is inversely proportional to the power loss between node $\mathrm{i}$ and $\mathrm{j}(\mathrm{a})$

$\mathrm{p}_{\mathrm{ij}}^{\mathrm{k}}=\eta_{\mathrm{ij}} \alpha / \mathrm{P}_{\text {lossij }}$

Therefore the probabilistic function is expressed as follows:

$$
\begin{aligned}
\mathrm{P}_{\mathrm{mj}}{ }^{\mathrm{k}}(\mathrm{t})=\left[\mathrm{T}_{\mathrm{mj}}(\mathrm{t})\right]^{\alpha *}\left[\dot{\eta}_{\mathrm{mj}}\right]^{\beta} / \Sigma\left[\mathrm{T}_{\mathrm{mk}}(\mathrm{t})\right]^{\alpha *}\left[\dot{\eta}_{\mathrm{mk}}\right]^{\beta} & ; \text { if jé allowed; and k } \varepsilon \text { allowed } \\
=0 & ; \text { otherwise }
\end{aligned}
$$




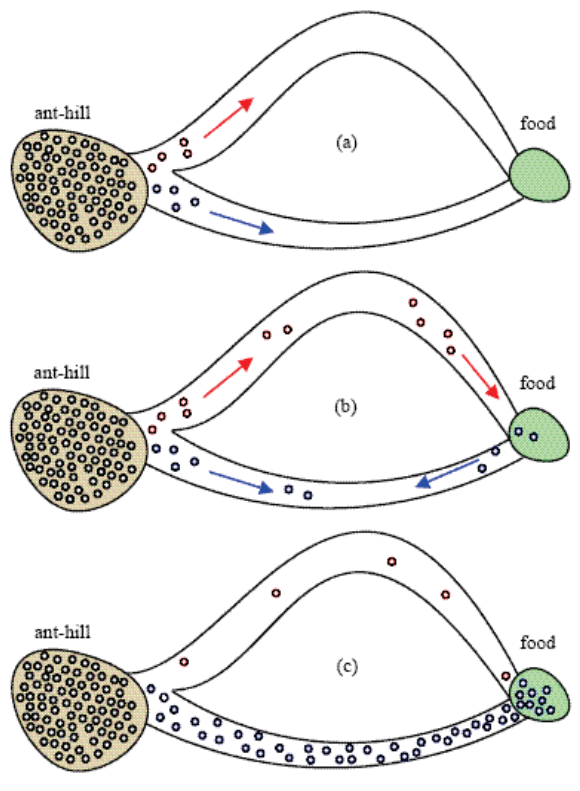

Fig. 1 Ants optimizing the food winning

Constraints:

(i) Operating voltage at each node must be in its safety range

$\mathrm{V}_{\mathrm{i}}^{\min }<=\mathrm{V}<=\mathrm{V}_{\mathrm{i}}^{\max }$

(ii) The Power flow at each node must be at balance and power flow at each branch must be less than or equal to its maximum capacity (a)

$\mathrm{S}_{\mathrm{i}}<=\mathrm{S}_{\mathrm{i}}^{\max }$

(iii) Load center (node) must not be isolated without supply from any feeder.

The algorithm developed is as follows:

Step 1: Initialization. Set time, $t=0 ;$ Cycles $N C_{\max }=10$;

Pheromone intensity in each branch is set initially to a constant value and also the change in pheromone intensity is set to zero $T_{i j}(0)=0.01$, $\Delta T_{i j}=0, n=m=14, \alpha=0.1, \beta=2, \rho=0.1$

where

$\mathrm{t}=$ time

$\mathrm{NC}=$ number of cycles

$\mathrm{T}_{\mathrm{ij}}=$ pheromone intensity

$\Delta \mathrm{T}_{\mathrm{ij}}=$ change in pheromone intensity

$\mathrm{m}=$ number of ants

$\mathrm{n}=$ number $\mathrm{f}$ nodes

$\alpha, \beta, \rho=$ constants

Step 2: Obtain the number of nodes and place $m$ ants on $n$ nodes and store the initial node of each ant in the Tabu list (i.e.) Tabu $(1, m)$ for all $m$ ants

Step 3: Set a loop for $m=1$ to 14

Calculate Power loss between two nodes $m \& j$ using the formula
$P_{\text {loss }}=\left(r_{m-j}\right)\left[\left(P_{j}^{2}+Q_{j}^{2}\right) /\left(V_{j}^{2}\right)\right]$

Here $\mathrm{j}$ denotes all the nodes directly connected to node $\mathrm{n}$ on which $\mathrm{m}$ ant is positioned.

Step 4: Calculate the probability for the ants to move to the next node for all possible routes. The probability of transition of ants from node $m$ to node $j$ is given by the following condition

$\boldsymbol{I} \boldsymbol{f}<j$ belongs to the set of nodes not yet visited by the ant $>$

Then $<P_{m j}{ }^{k}(t)=\left[T_{m j}(t)\right]^{\alpha *}\left[\dot{\eta}_{m j}\right]^{\beta} / \Sigma\left[T_{m k}(t)\right]^{\alpha *}\left[\dot{\eta}_{m k}\right]^{\beta}>$

Else $<0>$

And $\eta=1 / \mathrm{P}_{\text {lossmj }}$

Enter the node $j$ which is the transition of the ant from node in the second row of the Tabu list. (i.e.) in Tabu $(2, k)$ similarly, transition is made for all the nodes. Thus we will have 14 tours generated by 14 ants.

Step 5: Calculate the losses occurring for each tour and store it in an array

Step 6: Based on these 14 tours, find the branches, which occur the least number of times in all the 14 tours because they will be the branches with minimum probability or maximum losses. Arrange the branches in order of increasing transmission losses.

Step 7: The intensity of pheromone in each branch is updated using Global Pheromone Revision Rule as given below

$T_{m j=}(1-\alpha) \cdot T_{m j}+\Sigma \Delta T_{k}(i, j)$

where $\Delta T_{k}(i, j)=1 / P_{\text {lossk }} \quad$ (if $(i, j)$ comes in the tour generated by that particular ant)

$$
=0 \quad \text { :otherwise }
$$

Step 8: If the number of cycle is less than the maximum number of cycles $\left(N C<N C_{\text {max }}\right)$ then, clear the Tabu list, go to step 2 otherwise go to next step.

Step 9: stop

\section{Implementation \& Simulation}

IEEE 14- bus system is taken as the network on which the validity of the ant colony system algorithm for network reconfiguration is tested. The test system as shown in Fig. 2 consists of the slack bus (node 1), three PV (nodes 2, 3, and 6), ten PQ buses, and 20 branches. It has been used in many probabilistic studies. They comprise six discrete distributions for the active load (at nodes 3, 6, 9, 10, 11 , and 14), four discrete distributions for the reactive load (at nodes 9, 10, 11, and 14), with three to five impulses each and eight normal distributions for active and reactive loads at the remaining buses. The bus voltage is set to $0.44 \mathrm{KV}$. 


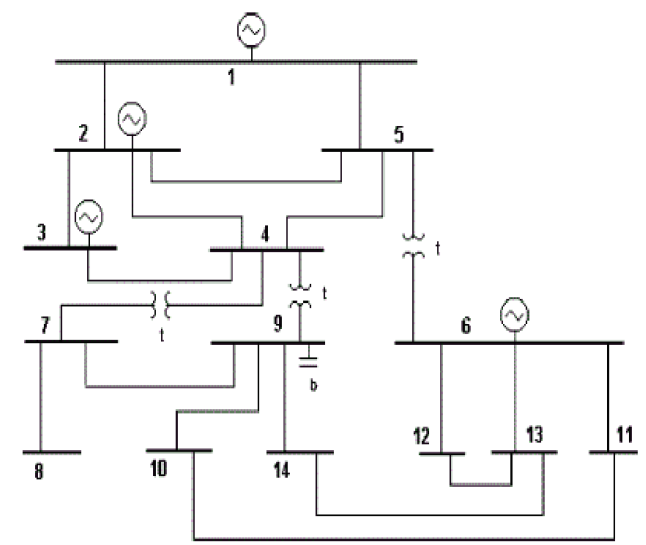

Fig. 2 IEEE 14 bus system

\section{Simulation Results}

The entire network is simulated in E-TAP software. E-TAP software is the most comprehensive analysis platform for design as well as simulation of generation, distribution and industrial power systems. In this work, this software is mainly used for performing the load flow analysis of the electrical network. It is found that when a fault occurs on line 2-4, its neighboring lines 2-3 and 2-5 get overloaded and also other electrical constraints are violated. Losses of $375 \mathrm{~kW}$ are found to be occurring in the network. Therefore, it is required to reconfigure the network in such a way that all the losses are minimized, overloaded lines are relieved and electrical constraints are maintained in their defined limits. Now the proposed ant colony algorithm which has been coded using $\mathrm{C}$ language is used to find out the lines which present minimum losses. Based on this data, line 5-4 is opened as doing so does not violate any electrical constraints and also the overloads are relieved. The losses are reduced to $318 \mathrm{~kW}$. Thus, we find the ant colony system based network reconfiguration gives a loss reduction of nearly $11 \%$.

Table 2 Simulation Results of IEEE-14 Bus System

\begin{tabular}{|l|l|l|}
\hline S. No. & Method Used & $\begin{array}{l}\text { Power Loss } \\
\text { Reduction (\%) }\end{array}$ \\
\hline 1. & $\begin{array}{l}\text { Artificial Bee Colony } \\
\text { Algorithm [29] }\end{array}$ & 8.15 \\
\hline 2. & SA [30] & 8.86 \\
\hline 3. & DE [31] & 8.86 \\
\hline 4. & Proposed Method & 11 \\
\hline 5. & GA [32] & 8.85 \\
\hline
\end{tabular}

For validation the proposed method is compared with other method in percentage of power loss reduction. From the Table 2, it is observed that the optimal power loss obtained by the proposed method is less than the artificial bee colony algorithm, simulated annealing technique, hybrid differential evolution \& genetic algorithm technique, as stated in table.

To further test the validity of the proposed algorithm, it is implemented on a 16-bus network. A Modified Genetic Algorithm for distribution network reconfiguration has been proposed (Radha, King, and Rughooputh, 2003). The results of this algorithm when applied to a 16- bus network showed a loss reduction of $6 \%$. When Ant colony system algorithm was applied to the same network, a loss reduction of nearly $10 \%$ was obtained. Thus the algorithm is found to be an efficient tool for network reconfiguration.

\section{Conclusion}

A new methodology, based upon the Ant Colony System (ACS) algorithm, is proposed for the reconfiguration of electric energy distribution systems. The methodology is very flexible and finds the optimal network with lower transmission losses while enforcing the technical constraints such as the transmission capabilities and the limits on voltage magnitudes.

The algorithm is coded in $\mathrm{C}$ language and further results are calculated using E-Tap software. Test results show that the ACS algorithm can be used to find optimum solutions within a reasonable time. The ACS methodology has the following characteristics like Positive feedback, Distributed computation, Greedy heuristic which makes the ACS algorithm to be the best suitable method for network reconfiguration. Since the algorithm involves a probability based search, the decision for the ants is made simple. Computer simulations are run for ACS algorithm which generates optimal network reconfiguration.

This algorithm can be applied and results can be verified for larger real time networks. In order to increase the speed of convergence, an evolutionary algorithm, such as the cultural-ACS algorithm, can be introduced in the future to determine the optimum values of the empirical parameters of the ACS algorithm.

\section{References}

1. L. Charles Daniel, I. Hafeezulah Khan, S. Ravichandran, Distribution Network Reconfiguration for Loss Reduction Using Ant Colony System Algorithm, in Proc. Of Indicon (IEEE, Chennai, 2005), pp. 619-622. 
2. Radha Bhoomesh, T. F. Ah King Robert, C. S. Rughooputh Harry, Optimal Network Reconfiguration of Electrical Distribution Systems, in Proc. Of ICIT (IEEE, Maribor, 2003), pp. 66-70.

3. S. Civanlar, J. J. Grainger, H. Yin, S. S. H. Lee, Distribution feeder reconfiguration for loss reduction, IEEE Transactions on Power Delivery (3) (1988) 1217-1223.

4. M. E. Baran, F. F. Wu, Network reconfiguration in distribution systems for loss reduction and load balancing, IEEE Transactions on Power Delivery (4) (1989) 1401-1407.

5. K. Nara, A. Shiose, M. Kitagawoa, T. Ishihara, Implementation of genetic algorithm for distribution systems loss minimum reconfiguration, IEEE Trans. Power Syst.(7) (1992) 1044-1051.

6. S. K. Goswami, S. K. Basu, A new algorithm for the reconfiguration of distribution feeders for loss minimization, IEEE Trans. Power Delivery (7) (1992) 1484-1491.

7. D. Shirmohammadi, H. W. Hong, Reconfiguration of electric distribution networks for resistive line loss reduction, IEEE Transaction on Power Delivery (4) (1989) 1492-1498.

8. T. P. Wagner, A. Y. Chikhani, R. Hackam, Feeder reconfiguration for loss reduction: An application of distribution automation, IEEE Transactions on Power Delivery (6) (1991) 1922-1931.

9. H. C. Cheng, C. C. Kou, Network reconfiguration in distribution systems using simulated annealing, IEEE Transactions on Power Delivery (29) (1994) 227-238.

10. H. D. Chiang, J. J. Rene, Optimal network reconfiguration in distribution systems-Part 1: A new formulation and a solution methodology, IEEE Transaction on Power Delivery (5) (1990) 19021908.

11. R. P. Broadwater, A. H. Khan, H. E. Shaalan, R. E. Lee, Time varying load analysis to reduce distribution losses through reconfiguration, IEEE Transaction on Power Delivery (8) (1993) 294-300.

12. S. Jazebi, S.H. Hosseinian, M. Pooyan, B. Vahidi, Performance comparison of GA and DEA in solving distribution system reconfiguration problem, in Proc. Of Int. Conf. on Optimization of Electrical and Electronic Equipment (IEEE, Brasov, 2008) pp. 185190.

13. R. Storn, K. Price, Minimizing the real functions of the ICEC'96 contest by differential evolution, Proceedings of Evol. Comput. Conf., (IEEE, 1996) pp. 842-844.

14. K. V. Price, Differential evolution vs. the functions of the 2nd ICEO, Proceedings of Evol. Comput. Conf., (IEEE, 1997) pp. 153-157.

15. R. Storn, System design by constraint adaptation and differential evolution, IEEE Transactions on Evol. Comput. (3) (1999) 22-34.

16. Belkacem Mahdad, Optimal Location and Control of Multi Hybrid Model Based Wind-Shunt FACT to Enhance Power Quality in Electrical Generation and Distribution Systems and Power Quality Disturbances (InTech, 2011).

17. J. P. Chiou, F. S. Wang, A hybrid method of differential evolution with application to optimal control problems of a bioprocess system, Proceedings of Evol. Comput. Conf., (IEEE, 1998) 627-632.

18. Y. C. Lin, F. S. Wang, K. S. Hwang, A hybrid method of evolutionary algorithms for mixed-integer nonlinear optimization problems, Proceedings of IEEE Evol. Comput. Conf., (IEEE, 1999) 21592166.

19. Y. C. Lin, K. S. Hwang, F. S. Wang, Plant scheduling and planning using mixed-integer hybrid differential evolution, solution with multiplier updating, Proceedings of IEEE Evol. Comput. Conf., (IEEE, 2000) 593-600.

20. M. Dorigo and T. Stutzle, Ant Colony Optimization (MIT Press, Cambridge, MA, 2004).

21. M. Dorigo, G. Di Caro, and L. M. Gambardella, Ant algorithms for distributed discrete optimization, Artificial Life, 5(2) (1999) 137-172.

22. Y. H. Song, G. S. Wang, Distribution network reconfiguration for loss reduction using fuzzy controlled evolutionary programming, IEE Proceedings on Generation, Transmission, Distribution, 144(4)(1997) 345-350.

23. N. Gupta, A. Swarnkar, K.R. Niazi, R.C. Bansal, Multi-objective reconfiguration of distribution systems using adaptive genetic algorithmin fuzzy framework, IET Gener. Transm. Distrib., 2010, Vol. 4, Iss. 12, (2010)1288-1298.

24. A.C. Santos, A. C. B. Delbem, Node-Depth Encoding and Multiobjective Evolutionary Algorithm Applied to Large-Scale Distribution System Reconfiguration, IEEE Transaction on Power Systems (25) (2010) 1254-1265.

25. Helon David de Macêdo Braz, Benemar Alencar de Souza, Distribution Network Reconfiguration Using Genetic Algorithms With Sequential Encoding: Subtractive and Additive Approaches, IEEE Transaction on Power Systems (26) (2011) 582- 593.

26. Rayapudi Srinivasa Rao, Sadhu Venkata Lakshmi Narasimham, Manyala Ramalinga Raju, A.Srinivasa Rao, Optimal Network Reconfiguration of LargeScale Distribution System Using Harmony Search Algorithm, IEEE Transaction on Power Systems (26) (2011) 1080 - 1088.

27. B. Amanulla, Saikat Chakrabarti, S. N. Singh, Reconfiguration of Power Distribution Systems

28. Considering Reliability and Power Loss, IEEE Transaction on Power Delivery (27) (2012) 910 $-926$.

29. R.Muthu Kumar, K.Thanushkodi, Network Reconfiguration And Restoration in Distribution Systems through Oppostion based Differential Evolution Algorithm And Pgsa, International Conference on Current Trends in Engineering and Technology (2013), $284-290$.

30. R. Srinivasa Rao, S.V.L. Narasimham, M. Ramalingaraju, Optimization of Distribution Network Configuration for Loss Reduction Using Artificial Bee Colony Algorithm, World Academy of Science, Engineering and Technology (2008),

31. $708-714$.

32. H. C. Cheng and C. C. Kou, Network reconfiguration in distribution systems using simulated annealing, Elect. Power Syst. Res., vol. 29, 227-238, May 1994.

33. C. T. Su and C. S. Lee, Network reconfiguration of distribution systems using improved mixed-integer hybrid differential evolution, IEEE Trans. on Power Delivery, Vol. 18, No. 3, July 2003.

34. Marcos A. N. Guimaraes, Carlos A. Castro, Ruben Romero, Reconfiguration of distribution systems by a modified genetic algorithm, in Proc. of PowerTech (IEEE, 2007) 1-5. 